\title{
As dimensões demográficas das mudanças climáticas: cenários de mudança do clima e as tendências do crescimento populacional
}

\author{
Ricardo Ojima*
}

\begin{abstract}
A mudança climática tem sido um tema cada vez mais presente no discurso político, econômico e social. É inegável que hoje tornou-se uma questão da mais ampla relevância para toda a sociedade, mas, especialmente, para a demografia. Como parte inerente dos cenários de mudança climática, a melhor compreensão da dinâmica demográfica nesse contexto tem muito a contribuir para um debate responsável. Entretanto, ainda há lacunas a serem preenchidas dos dois lados: as ciências do clima e os estudos de população. Este artigo busca trazer elementos para deslocar o assunto da arena ambientalista para uma visão mais ampla do que poderia ser uma demografia das mudanças climáticas.
\end{abstract}

Palavras-chave: Projeções e tendências. Dinâmica demográfica. Mudança climática.

\section{Introdução}

Desde a publicação, em 2007, do 4 은 relatório do IPCC (Intergovernmental Panel on Climate Change), o AR-4, o debate ambiental obteve um destaque político, econômico e social que até então só poderia ser comparado à Conferência das Nações Unidas de 1992, a Rio 92. Passados quase 20 anos dessa conferência no Rio de Janeiro, o que temos em debate é muito mais do que uma discussão relacionada à poluição e degradação dos recursos naturais. A perspectiva da problemática ambiental hoje parece assumir um aspecto muito mais amplo e que efetivamente incorpora, à esfera da vida cotidiana, os desafios relacionados ao clima. Para Giddens (2010), há um deslocamento da discussão sobre mudanças climáticas para a corrente dominante e com isso potencializa-se uma diversidade maior de aproximações ao tema.
O debate que antes era exclusividade do movimento verde foi incorporado pelas mais diversas áreas, desde a economia até a política mais esquerdista, cada qual buscando se apropriar do tema à sua maneira e de acordo com seus interesses. Entretanto, a busca de uma ciência ou política da mudança climática não deve ser confundida com esses princípios "verdes". A preservação ou conservação que é um dos lemas do movimento verde tem pouca relação direta com as mudanças climáticas, se comparado com outros aspectos menos evidentes (GIDDENS, 2010, p. 80). Assim, como mencionado por Buttel et al. (2002), as questões ambientais tornam-se cada vez mais aspectos sociais do que meramente aqueles relacionados à natureza.

Neste contexto, é importante reiterar tal separação, pois, entre outras razões, essa percepção contribuiu para que tenham sido necessários 15 anos (entre o

\footnotetext{
* Sociólogo, doutor em Demografia, professor adjunto da Universidade Federal do Rio Grande do Norte - UFRN.
} 
primeiro e o quarto relatório do IPCC) para que as ciências humanas entrassem mais sistematicamente no debate sobre as mudanças climáticas. Hogan (2009) salienta que, até poucos anos atrás, era pequena a comunidade de pesquisadores que se debruçavam sobre as dimensões humanas das mudanças ambientais globais. A dimensão demográfica como era (e ainda é) desenvolvida no contexto da ciência das mudanças climáticas ainda não está totalmente relacionada como um fator social, devido, em parte, a esse atraso que, enfim, teve algumas justificativas e consequências.

As ciências do clima se pautam por padrões, tendências e séries históricas de dados para estimar e projetar cenários futuros. Em termos metodológicos, a consequência é um grau de análise de longo prazo em escalas espaciais mais abrangentes, na medida em que é necessário um conjunto de dados suficientemente grande para que os modelos sejam confiáveis. Por outro lado, as ciências humanas possuem uma escala espacial e temporal muito mais reduzida. Considerando-se a dinâmica demográfica um fator social, 100 anos pode ser uma escala temporal tão longa que os fatores de mudança social perdem sua capacidade explicativa. Um bom exemplo disso, levando-se em conta as mudanças demográficas brasileiras, é que as estimativas realizadas na década de 1970 apontavam para uma população de mais de 200 milhões de pessoas em 2000 , entretanto, tal cifra não foi atingida nem mesmo em 2010.

Este artigo procura inserir o fator populacional como uma variável social dentro da ciência das mudanças do clima, em busca de uma demografia das mudanças climáticas. Após uma breve revisão das previsões e cenários para as mudanças do clima e para o crescimento populacional, procura-se iniciar uma discussão sobre os pontos de convergência e tensão entre estes cenários e algumas das suas causas e consequências mais imediatas. Com isso, espera-se que este artigo traga elementos para se pensar o fator demográfico como um elemento social das mudanças climáticas e, com isso, colaborar em uma perspectiva integradora que vá além das leituras neomalthusianas que relacionam o volume populacional aos impactos ambientais.

\section{Clima}

Com a publicação do AR-4 e a demanda pela incorporação do tema das mudanças climáticas no discurso social, político e econômico, as ciências humanas e sociais se depararam com conceitos novos para seu desenvolvimento. Em parte, a abstração e as incertezas decorrentes dos modelos e hipóteses de aquecimento global até esse momento não haviam colocado tanta urgência para que os estudos sociais entrassem no debate. Mas o AR-4 consolidou o desenvolvimento do campo de pesquisas sobre o clima global de forma irrefutável, mostrando que algumas das consequências sociais esperadas já não podiam mais ser entendidas como meras especulações de ficção científica (HOGAN, 2009).

Criado no final de década de 1980 , o Painel Intergovernamental sobre Mudanças Climáticas (IPCC, na sigla em inglês) não é um grupo de pesquisa, mas sim um corpo internacional de especialistas para avaliação da pesquisa sobre mudanças climáticas. Assim, seu principal objetivo é promover uma visão ampla sobre o estado-da-arte a respeito da pesquisa realizada sobre mudança climática no mundo, consolidando e confirmando as tendências do campo de conhecimento e considerando seus impactos ambientais e socioeconômicos.

Por essa razão é que, embora haja ainda vozes dissonantes em relação à aceitação da interferência humana nas tendências de aquecimento global (MARANDOLA Jr., 2009), muitas conclusões trazidas pelo AR-4 são inequívocas. O Grupo de Trabalho I (GT-1/AR-4) descreve os avanços baseados nos relatórios anteriores do IPCC e aqueles obtidos ao partir do terceiro relatório (AR-3) em relação à base das ciências físicas. $O$ sumário dos principais resultados do GT-I, assim como os demais sumários para formuladores de políticas públicas (IPCC, 2007a), considera apenas os resultados com nível de confiança muito alto e alto.

O tratamento da incerteza, como mencionado, é um dos elementos que avançam 
significativamente desde o terceiro relatório. De forma geral, a incerteza é medida de três formas diferentes no AR-4. Quando as informações trazem uma avaliação baseada em análises estatísticas, são apresentadas escalas de probabilidade variando de $100 \%$ (virtualmente certo) até $<1 \%$, como excepcionalmente pouco provável. Nos casos em que a avaliação é feita de modo quantitativo, mas sem análises estatísticas, a incerteza é medida conforme o julgamento dos especialistas da área, baseados nas evidências, variando de zero a 10, conforme a sua avaliação de que a afirmação esteja correta. Particularmente no Grupo de Trabalho III (GT-III), a avaliação é baseada em apreciação qualitativa e é mensurada basicamente pelo grau de concordância entre o conjunto das pesquisas avaliadas. Assim, os termos usados para medir a incerteza são "alta concordância", "alta evidência", etc. (IPCC, 2007d).

Mas, embora muitas vezes os termos se confundam, as mudanças climáticas não têm necessariamente uma relação direta com mudanças ambientais. De fato, as primeiras são consequência de mudanças no ambiente, mas também exercem influência sobre ele. O clima é um sistema complexo e interage com a atmosfera, superfície, geleiras, oceanos, corpos d'água e seres vivos e, por isso, o seu estudo depende da compreensão de um conjunto grande de variáveis que interagem entre si (IPCC, 2007a). Neste sentido, um dos mais importantes componentes dos esforços do IPCC é, portanto, o desenvolvimento de cenários do potencial futuro do fator antropogênico, considerando as forças motrizes e as opções de resposta às mudanças climáticas (MOSS et al., 2008).

Segundo o GT-III/AR-4 (IPCC, 2007a), as informações paleoclimáticas ${ }^{1}$ confirmam que o aquecimento ocorrido nos últimos 50 anos foi incomum. Assim, é muito provável que as alterações no clima recente sejam reflexo do aumento observado nas concentrações de gases de efeito estufa (GEE), havendo, neste caso, uma contribuição significativa das atividades humanas ocorridas nesse período (IPCC, 2007a). As observações diretas das mudanças no clima confirmam que o período1995-2006 corresponde aos 12 anos mais quentes registrados desde 1850 . Somado a isso, a temperatura média do oceano global aumentou e absorveu mais de $80 \%$ do calor acrescentado ao sistema climático; assim, a expansão da água do mar teve um papel na elevação média do nível do mar a uma taxa média de 1,8 mm por ano, desde 1961 (IPCC, 2007a).

Com o avanço dos modelos computacionais de simulação de cenários de mudança do clima, o IPCC avaliou um conjunto de possibilidades, no Relatório Especial sobre Cenários de Emissões (Rece), de 2000. Considerando-se tais cenários, mesmo que as concentrações de GEE e aerosóis se mantivessem constantes nos níveis de 2000, seria esperado um aumento mínimo de 0,1 grau Celsius por década. Assim, as projeções para os próximos 100 anos seriam de um aquecimento entre 0,3 e 0,9 grau Celsius, mesmo que permanecessem os padrões de emissão de 2000 , o que, do ponto de vista empírico e observado atualmente, seria muito pouco provável tendo em vista os ritmos de crescimento econômico dos países asiáticos, como a China.

Considerando esse grau de incerteza, pode-se afirmar com relativa segurança que, não importando o que se faça a partir de agora para mitigar os fatores forçantes antropogênicos do clima, haverá uma mudança esperada para o século XXI, tais como dias e noites quentes mais frequentes, ondas de calor, eventos de precipitação extrema, aumento das áreas já afetadas pelas secas, entre outros (IPCC, 2007a). Mas, se não há o que fazer para evitar tais mudanças, como devemos agir? Não se trata de resgatar, portanto, a reverência mística pela natureza

\footnotetext{
1 Os estudos paleoclimáticos utilizam indicadores sensíveis ao clima para inferir mudanças passadas no clima global em escalas de tempo que podem ir até milhares de anos. Indicadores como a largura dos anéis das árvores ou os testemunhos de gelo nos polos, que são influenciados pela temperatura, precipitação, entre outros, permitem estimar o comportamento do clima mesmo quando informações diretas não existiam.
} 
TABELA 1

Projeção do aquecimento médio global da superfície e da elevação do nível do mar no final do século XXI, segundo cenários

\begin{tabular}{|c|c|c|c|}
\hline \multirow{2}{*}{ Cenários } & \multicolumn{2}{|c|}{$\begin{array}{l}\text { Mudança de temperatura }\left({ }^{\circ} \mathrm{C} \text { em }\right. \\
2090-2099 \text { em relação a } 1980-1999)(1)\end{array}$} & \multirow{2}{*}{$\begin{array}{c}\text { Elevação do nível do mar (metros em } \\
2090-2099 \text { em relação a 1980-1999) } \\
\text { Faixa com base em modelo, } \\
\text { excluindo-se as futuras mudanças } \\
\text { dinâmicas rápidas no fluxo de gelo }\end{array}$} \\
\hline & Melhor estimativa & Faixa provável & \\
\hline $\begin{array}{l}\text { Concentrações constantes } \\
\text { do ano de } 2000(2)\end{array}$ & 0,6 & $0,3-0,9$ & Não disponível \\
\hline Cenário B1 & 1,8 & $1,1-2,9$ & $0,18-0,38$ \\
\hline Cenário A1T & 2,4 & $1,4-3,8$ & $0,20-0,45$ \\
\hline Cenário B2 & 2,4 & $1,4-3,8$ & $0,20-0,43$ \\
\hline Cenário A1B & 2,8 & $1,7-4,4$ & $0,21-0,48$ \\
\hline Cenário A2 & 3,4 & $2,0-5,4$ & $0,23-0,51$ \\
\hline Cenário A1FI & 4,0 & $2,4-6,4$ & $0,26-0,59$ \\
\hline
\end{tabular}

Fonte: IPCC (2007a).

(1) Essas estimativas são avaliadas a partir de uma hierarquia de modelos que abrangem um modelo climático simples, vários EMICs (Earth System Models of Intermediate Complexity - Modelos do Sistema Terrestre de Complexidade Intermediária) e um grande número de AOGCMs (Atmosphere-Ocean Circulation Models - Modelos de Circulação Geral da Atmosfera-Oceano).

(2) A composição constante do ano 2000 é derivada apenas a partir dos AOGCMs.

herdada do movimento verde, já que a forma de lidar com o aquecimento global nada tem a ver com valores altruístas para salvar o planeta da devastação do homem, sendo que tais tendências ocorrerão de forma mais ou menos incisiva de acordo com o que fizermos, mas, de uma forma ou de outra, o planeta vai continuar existindo (GIDDENS, 2010, p. 81).

Como é feito pelas ciências do clima, é importante que se reconheçam e se investiguem os mecanismos demográficos que envolvem a variação do clima. É preciso abrir mão de valores morais e simplificações reducionistas das causas e efeitos antropogênicos. Afinal, já é certo que o fator humano é um dos principais elementos a serem considerados nos modelos climáticos. Talvez a dificuldade de articular o que se pode projetar a partir desses cenários de clima para o futuro e as medidas efetivas de mitigação e adaptação esteja no fato de se desconsiderarem os elementos sociais e políticos envolvidos no processo. Nesse sentido, o componente demográfico ainda é subutilizado nas análises de mudanças climáticas (HOGAN, 2009), mesmo que sejam reconhecidas as contribuições mais impactantes de outros aspectos demográficos para os níveis de emissões (LUTZ;
PRSKAWETZ; SANDERSON, 2002; O'NEILL; MACKELLAR; LUTZ, 2001).

Em verdade, a sobreposição do período de desenvolvimento econômico como principal fator antropogênico forçante para as mudanças climáticas com o período de elevado crescimento demográfico é um dos fatores que dificulta a análise mais cuidadosa dessa relação. Dessa maneira, a facilidade de se reduzir a questão demográfica no contexto da mudança climática a apenas uma questão volumétrica é grande e até esperada. Assim, embora entre os demógrafos essa questão já tenha sido superada há muito tempo, ainda há um abismo grande entre os dois lados da equação, devido, principalmente, à necessidade de se avançar nos estudos que toquem nas variáveis não imediatas do crescimento demográfico.

\section{Crescimento populacional}

Quando pensamos historicamente no século $X X$, não há como não pensar nas grandes mudanças que o marcaram. Mudanças rápidas e significativas em diversas esferas da vida social ocorreram em um breve período de tempo, que, como argumenta Hobsbawn (1994), pode ser lembrado como um século curto, marca- 
do por transformações extremas. Nesse contexto, como separar as várias variáveis que se sobrepõem e como entender os processos que interagem entre si de maneira independente e desprendida de vícios de análise? Já foi mencionado que a principal elevação da temperatura média registrada desde 1850 ocorreu na última década deste século. Foi também na segunda metade do século $X X$ que a concentração atmosférica de dióxido de carbono apresentou uma taxa de aumento de concentração mais elevada (1,4 parte por milhão, média de 1960 a 2005) (IPCC, 2007a).

Também durante o século XX o mundo experimentou o mais rápido crescimento demográfico da história, passando de 1,65 bilhão para 6 bilhões de pessoas, registrando o maior pico na taxa média de crescimento anual ( $2 \%$ ao ano) e o maior incremento anual de pessoas por ano (86 milhões ao ano), nas décadas de 1960 e 1980, respectivamente (Gráfico 1). Coincidentemente, foi na segunda metade deste século que as taxas de crescimento populacional apresentaram ritmo mais acelerado, o que, de certa forma, deixa virtualmente vinculados o aumento de emissões de GEE e a expansão populacional.

Os cenários avaliados pelo IPCC consideram o crescimento populacional um dos fatores forçantes, entretanto, cada cenário leva em conta uma tendência de crescimento diferente. Isso, segundo Zlotnik (2009), torna difícil a análise detalhada do impacto real que o componente demográfico tem nos modelos avaliados. Mas essa característica permite confirmar o fato de que, a despeito das tendências demográficas observadas, o fator demográfico utilizado para as proje-

GRÁFICO 1

Evolução da população mundial e estimativas (1)

1750-2050

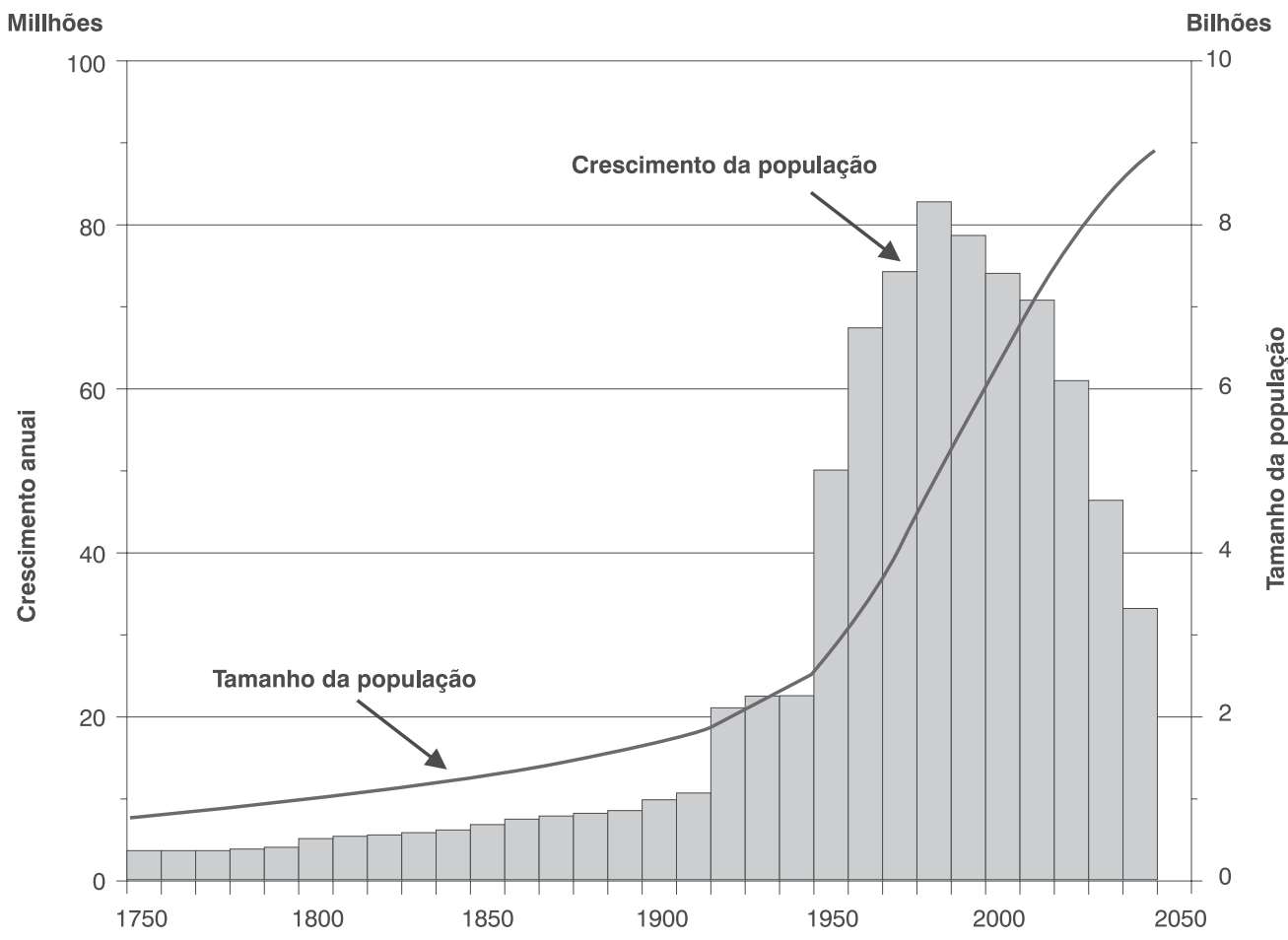

Fonte: United Nations, 1999

(1) Revisão de 1998. 
ções do clima considera o crescimento demográfico o único fator forçante do aumento das emissões de GEE.

De fato, considerando o padrão dos dias atuais, quanto maior for o número de pessoas no mundo, maiores seriam as emissões de GEE derivadas. O cenário A2, um dos que apresentam maiores impactos decorrentes de mudanças climáticas, é, entre outros elementos, aquele que combina elevado crescimento populacional (15 bilhões de pessoas em 2100) com poucas mudanças tecnológicas. Os cenários $\mathrm{A} 1$ e B1, mais contidos em termos de impactos de mudanças climáticas, são também aqueles em que as variantes do crescimento populacional são as mais baixas, considerando um pico de 8,7 bilhões de pessoas em 2050 e uma redução para 7,1 bilhões até 2100 (ZLOTNIC, 2009, p. 35).

Entre os demógrafos, há consenso em relação às tendências do crescimento populacional, que, para os não especialistas, talvez pareçam conter o mesmo grau de incerteza daquele encontrado em relação às estimativas para o crescimento econômico ou aos níveis de emissões de GEE. O ritmo de crescimento demográfico elevado observado principalmente na segunda metade do século XX foi um fenômeno excepcional na trajetória demográfica e está relacionado a fatores muito claros, quando se pensa nos componentes da dinâmica demográfica. Este crescimento demográfico excepcional está relacionado ao processo de transição demográfica, particularmente no momento intermediário em que as taxas de natalidade e mortalidade estão passando dos níveis altos de equilíbrio para baixos.

O Gráfico 2 mostra como essa transição afeta o incremento populacional, sobretudo a partir do final do século XX. Com a redução dos níveis de mortalidade, em parte associados a melhorias em saneamento básico e avanços na medicina, os níveis de natalidade, que ainda eram elevados, contribuíram para que o crescimento populacional apresentasse taxas elevadas por um período de tempo muito curto. Mas, ao contrário do que o público não especializado espera, as tendências do crescimento populacional são mais seguras e confiáveis do que aquelas referentes aos padrões de emissões de GEE ou ao ritmo de crescimento econômico (MARTINE, 2009).

As projeções realizadas pela Organização das Nações Unidas (ONU), em 2008, apontam que a população mundial deverá atingir a marca de 9,1 bilhões de pessoas em 2050, considerando a variante média. Essa variação de cerca de 33 milhões de pessoas adicionais por ano, até 2050 , dependerá fortemente da confirmação das hipóteses de fecundidade previstas para o futuro, que consideram que a taxa passará de uma média atual de 2,56 filhos por mulher para 2,02, em 2050 (UNITED NATIONS, 2009). Essa estimativa do número médio de filhos por mulher leva em conta, portanto, uma tendência observada nas últimas décadas de declínio gradual no ritmo médio de nascimentos, apesar de ainda se observar crescimento demográfico absoluto.

Isso ocorre devido a uma inércia relativa no comportamento demográfico, em que as experiências do padrão de nascimentos e óbitos ainda permanecem exercendo influência sobre o volume total da população. Assim, mesmo que se atinja em 2050 (como é previsto pelas projeções das Nações Unidas) uma taxa de reposição da população em torno de 2 filhos por mulher, ainda haverá crescimento populacional relativamente elevado derivado do número maior de muIheres nascidas sob a experiência de mais elevadas taxas de fecundidade no passado (CARVALHO, 2004; CARVALHO; GARCIA, 2003; CARVALHO; WONG, 2008).

Assim, espera-se que o ritmo de crescimento demográfico diminua gradativamente a partir de 2050, quando se atingirá o nível de reposição da população. Claro que, em estimativas de muito longo prazo, pequenas variações nas hipóteses assumidas poderão trazer mudanças significativas no volume populacional, entretanto, assumindo as tendências recentes, pode-se dizer que mesmo as hipóteses mais conservadoras têm se mostrado acima do esperado em estimativas passadas. Ou seja, é muito provável que a estimativa média assumida pelas Nações Unidas seja cumprida. Nesse sentido, pode-se esperar que, a partir de 2050, ocorra não apenas uma estabiliza- 


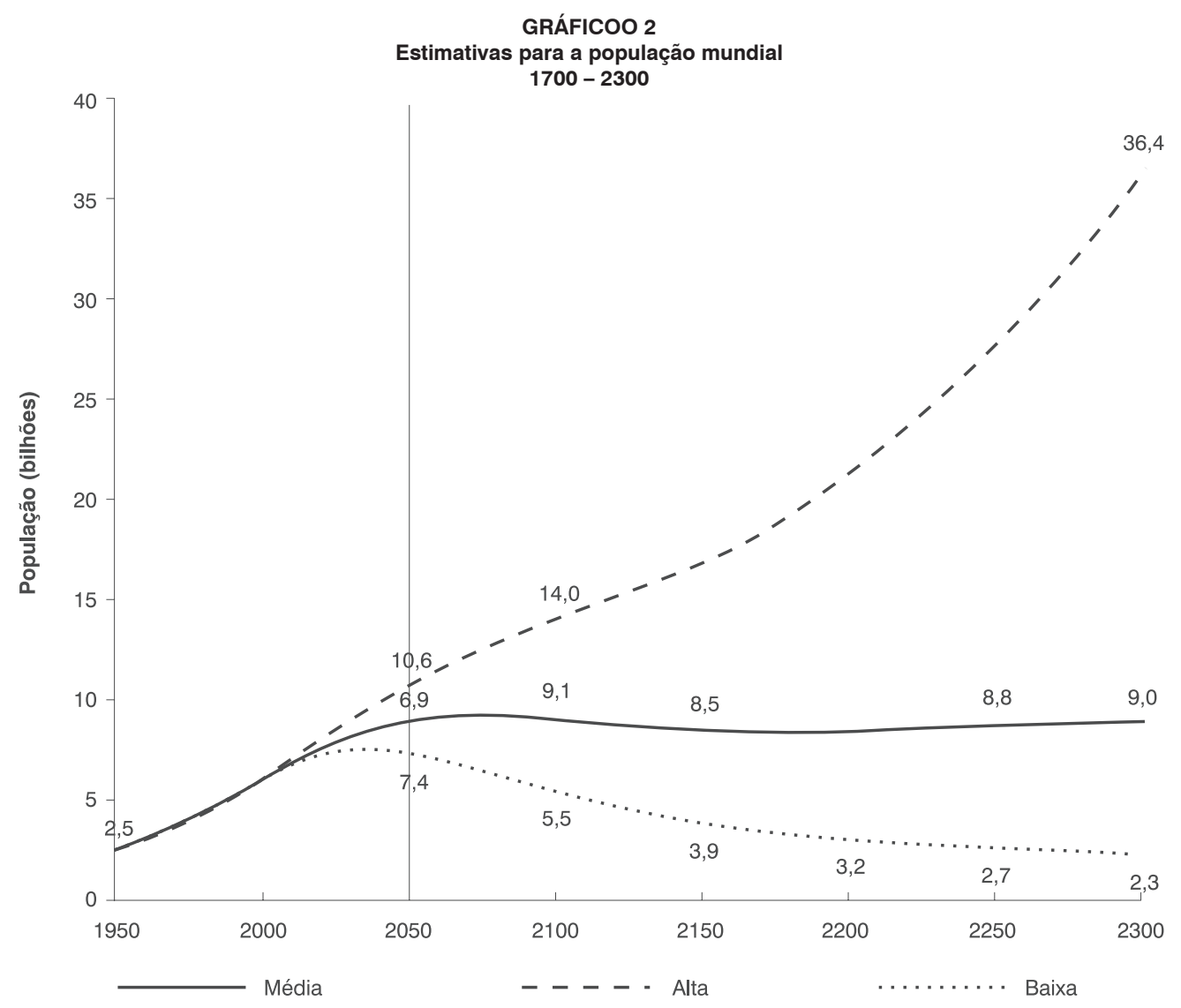

Fonte: United Nations (2004).

ção da população mundial, mas também uma perspectiva muito plausível de taxas de crescimento negativo, considerando a variante baixa das projeções das Nações Unidas para 2300 (UNITED NATIONS, 2004).

Esse cenário de estabilização da população mundial em torno dos 9 bilhões de pessoas trará elementos novos para se pensar nos forçantes das mudanças climáticas. Do ponto de vista demográfico, essa estabilização do crescimento populacional e uma eventual taxa de crescimento negativo da população, como é esperado ocorrer em diversas regiões e países do mundo, terão consequências importantes na estrutura etária e em outros aspectos sociais dessas populações. Isso já vem ocorrendo em algumas regiões que passaram pelo processo de transição demográfica mais precocemente, como a Europa e, particularmente, a América Latina, que passou pela transição mais recentemente e em uma escala temporal muito mais breve.

Assim, se o crescimento populacional for considerado um forçante negativo para as mudanças climáticas, pode-se dizer que, segundo as estimativas, em um prazo de menos de 50 anos, ele não será mais um problema grave. Mas será que por isso não haverá mais questões demográficas envolvidas nas mudanças do clima? Se analisadas as taxas de crescimento populacional, mesmo nos dias de hoje, ainda elevadas, é possível perceber que esse crescimento ainda se dá entre os países em desenvolvimento, onde os níveis de 
emissões são praticamente insignificantes em relação aos países do Anexo $1 .^{2}$ Então, o uso do volume populacional como indicador de aumento de emissões deve ser tomado com cuidado, uma vez que cerca de $80 \%$ das emissões de GEE ocorrem entre os países que respondem por cerca de $20 \%$ da população mundial.

\section{Mudança climática e população}

Como visto, o componente populacional entra no debate das mudanças climáticas por duas vias principais. Uma delas, a mais evidente e estudada, refere-se aos impactos e à vulnerabilidade dos grupos populacionais expostos às mudanças no clima. Como apontado por Giddens (2010), entender a vulnerabilidade para adotar medidas de adaptação de maneira pró-ativa é uma das preocupações urgentes para enfrentar os problemas que serão ampliados e potencializados pelas mudanças no clima.

Mas essa abordagem não é propriamente nova na demografia. Em verdade, o fator populacional já estava vinculado aos estudos ambientais sob essa perspectiva desde algum tempo. Os estudos de vulnerabilidade permitem um olhar mais amplo, entendendo a sua multidimensionalidade $e$ garantindo a compreensão das diferentes formas e intensidades dos riscos e perigos para cada grupo populacional (LUTZ; PRSKAWETZ; SANDERSON, 2002; MARANDOLA Jr., 2006; MARANDOLA Jr.; HOGAN, 2009; HOGAN; MARANDOLA Jr., 2005; HOGAN, 2005).

As mudanças climáticas podem ser motivo de movimentos populacionais e redistribuição espacial da população em diversas escalas espaciais. Direta ou indiretamente, tais mudanças podem afetar o maior ou menor grau de vulnerabilidade de determinados grupos populacionais, condicionando uma redistribuição espacial da população nestes contextos (BARBIERI et al., 2010; CARMO; SILVA, 2009). Os impactos na saúde humana também são aspectos da vulnerabilidade sociodemográfica que vêm sendo investigados no Brasil (CONFALONIERI; MARINHO; RODRIGUEZ, 2009), o que mostra que muitas pesquisas têm se concentrado na relação População-Mudança Climática recentemente.

O Grupo de Trabalho II (GT-II/AR-4) concentra-se nas avaliações de grupos populacionais vulneráveis e das medidas de adaptação consideradas. Em parte, uma das dificuldades de se confirmar com grau de confiança elevado os impactos já existentes das mudanças do clima está exatamente na capacidade de adaptação de grupos populacionais e regiões, o que dificulta sua confirmação, uma que impactos existentes podem já ter sofrido algum processo de adaptação (MCLEMAN, 2009; REID et al., 2007; MOSER; LUERS, 2008; KLEIN et al., 2007). Entretanto, apesar de haver muita pesquisa necessária nessa direção, esta é uma seara em que tradicionalmente a demografia não tem se furtado à análise. Mas, como apontado por Queiroz e Barbieri (2009), estudos empíricos sobre vulnerabilidade em relação a mudanças climáticas de mais longo prazo ainda são escassos, provavelmente pela dificuldade de projetar as interações das variáveis sociais, econômicas e demográficas.

De forma diferente, a outra entrada da dimensão demográfica sobre as mudanças climáticas ainda merece mais aprofundamento. Trata-se da incorporação da discussão demográfica enquanto fator forçante para os cenários de mudança do clima. Como mencionado, os cenários avaliados pelo IPCC para o aquecimento global incorporam o crescimento demográfico como um elemento forçante, em que um maior volume populacional estaria relacionado a um aumento nas emissões de GEE.

Assim, a ausência de uma contribuição efetivamente demográfica ao debate desconsidera a estrutura e composição da população como elementos forçantes ou mitigadores. Afinal, em quaisquer dos modelos usados para a construção de cenários de mudanças do clima, se reduzirmos o

\footnotetext{
2 Os países do Anexo 1 são signatários da Convenção Quadro das Nações Unidas para as Alterações Climáticas (UNFCCC). Entre eles estão Estados Unidos, Canadá, Japão, Austrália e alguns países do leste europeu.
} 
ritmo de crescimento demográfico, também diminuiremos os impactos das mudanças climáticas. Conclusão: o controle da natalidade vai mitigar os impactos das mudanças climáticas! E, após anos de desenvolvimento do campo de pesquisa, retornamos ao antigo dilema malthusiano?

O AR-4 claramente menciona que o Produto Interno Bruto (PIB) per capita e o crescimento populacional foram os principais condutores do aumento das emissões globais durante as últimas décadas do século XX. Mas, claro, quem seria a pessoa a sugerir uma redução no ritmo de crescimento econômico ou do aumento do PIB per capita? Somente os ambientalistas radicais teriam essa audácia. Assim, recai novamente sobre o ritmo de crescimento populacional a responsabilidade de reduzir o ímpeto das emissões de GEE.

O componente demográfico entra em cada um dos cenários avaliados pelo IPCC, no Relatório Especial de Cenários de Emissões (IPCC, 2000), em quatro combinações de mudança no desenvolvimento social e econômico e avanços tecnológicos (A1, A2, B1 e B2), sendo que, em cada um dos cenários, a projeção de crescimento demográfico é diferente e fortemente relacionada com o crescimento econômico. Os cenários são baseados em diferentes pressupostos de queda das taxas de fecundidade e de diferentes fontes.

Os cenários A1 e B1 incorporam as estimativas mais baixas baseadas em 1996, propostas pelo International Institute for Applied System Analysis (IIASA). Essa projeção considera baixas taxas de fecundidade para o início do século XXI, atingindo uma população global de 8,7 bilhões de pessoas em 2050, com declínio previsto para 2100 , chegando a cerca de 7 bilhões neste ano. No cenário B2, que emprega a projeção de longo prazo das Nações Unidas, com a variante média realizada em 1998, a população mundial atingiria 10,4 bilhões de pessoas em 2100 , sempre numa tendência crescente. E o cenário $A 2$ assume a variante alta das Nações Unidas, com uma população também crescente, mas atingindo 15 bilhões de pessoas em 2100 (IPCC, 2000).

Como qualquer cenário de projeção, as dificuldades de se imaginar como será o mundo nos próximos 100 anos são enormes, talvez nesse caso mais complexo do que estimar as taxas de fecundidade total, nas projeções demográficas (MARTINE, 2009). Assim, não bastaria extrapolar as tendências recentes de crescimento demográfico, desenvolvimento econômico e social e os avanços tecnológicos. Portanto, estes cenários são pensados em termos de linhas de história plausíveis, de modo que o cenário $A 1$ é o mais otimista, considerando as tendências do crescimento populacional e as demais variáveis relacionadas às mudanças climáticas. Além disso, o cenário A1 possui três subcenários, em que os avanços tecnológicos variam de acordo com uma economia intensiva em combustíveis fósseis (A1F1), baixo carbono (A1T) e um equilíbrio entre fontes de energia (A1B).

Apesar de incluírem projeções de diferentes fontes e com diferentes pressupostos, todos os cenários assumem uma relação de pressão demográfica sobre as emissões de GEE. Mas considerar o crescimento demográfico como forçante apenas do ponto

QUADRO 1

Principais pressupostos dos cenários de mudança climática do IPCC

\begin{tabular}{|c|c|c|c|c|}
\hline \multirow{2}{*}{ Ocorrências } & \multicolumn{4}{|c|}{ Cenários } \\
\hline & A1 & A2 & B1 & B2 \\
\hline Crescimento populacional & Baixo & Alto & Baixo & Médio \\
\hline Crescimento do PIB & Muito alto & Médio & Alto & Médio \\
\hline Mudança tecnológica & Rápido & Lento & Médio & Médio \\
\hline Uso de energia & Muito alto & Alto & Baixo & Médio \\
\hline Mudança de uso do solo & Baixo/Médio & Médio/Alto & Alto & Médio \\
\hline
\end{tabular}

Fonte: Adaptado de Jiang e Hardee (2009). 
de vista do seu crescimento é arriscado. A transição urbana, independentemente do aumento ou não do número de pessoas no globo, trará impacto no volume de emissões de GEE (MARTINE, 2009; OJIMA; HOGAN, 2009; EWING et al., 2008; SATTERHWAITE, 2009; DODMAN, 2009). A experiência de transição demográfica, associada à transição urbana ocorrida na América Latina e especialmente no Brasil, mostra como essa combinação vai além do volume da população para se pensar as tendências de emissões de GEE e do próprio modelo de urbanização esperado para o futuro (OJIMA, 2009).

Assim, será que o uso do estoque populacional é suficiente para estimar maiores impactos nas emissões de GEE? Considerando uma média per capita dos padrões médios atuais de emissões, sim. Dizer que uma pessoa adicional com um padrão de consumo médio global traria mais impactos do que se essa pessoa adicional fosse evitada não parece coerente? Esse é o proposto adotado com base nos cenários construídos pelo IPCC. Mas, levando-se em conta as projeções demográficas dos cenários $A 1$ e B1, de crescimento populacional baixo e com redução do estoque populacional até 2100 , quais seriam os impactos resultantes da mudança na estrutura etária, do processo de urbanização ou da redução do tamanho médio dos domicílios?

\section{Por uma demografia das mudanças climáticas}

Como visto anteriormente, embora muitas pesquisas já estejam incorporando as mudanças climáticas para antecipar o aumento de vulnerabilidades e impactos demográficos em cenários futuros, os estudos só começam a caminhar no sentido de incluir aspectos da dinâmica demográfica como fatores forçantes para as mudanças climáticas em meados da década de 1990. Mackellar et al. (1995), em um dos primeiros trabalhos relacionando a estrutura demográfica com o padrão de emissões, afirmam que a redução no tamanho dos domicílios, normalmente associada à queda da fecundidade, pode aumentar significativamente o padrão de emissões de GEE. Assim, um divórcio teria mais impactos nessas emissões do que um nascimento (UNFPA, 2009).

Segundo a pesquisa de Yu e Liu (2007), o divórcio teria um impacto de aumento de consumo, pois, com o desmembramento dos domicílios, "duplica" os itens de consumo, como geladeira, televisão, etc. Além disso, segundo os autores, os domicílios de divorciados são mais intensivos em consumo energético do que aqueles de casados, pois consomem $46 \%$ e $56 \%$ mais energia elétrica e água, respectivamente. Isso decorre do fato de que a dinâmica do consumo estaria mais fortemente relacionada à estrutura doméstica do que o volume populacional (CURRAN; DE SHERBININ, 2004; MacKELLAR et al., 1995).

Para Martine (2009), assim como para grande parte dos estudos que relacionam o tamanho do domicílio ao padrão de emissões de GEE nos países desenvolvidos, essa relação pode estar representando mais o efeito "consumo" do que o efeito demográfico. Assim, os países centrais (com baixas taxas de crescimento populacional) não apenas são os que mais contribuem com seu padrão de consumo elevado, mas também os que possuem arranjos domésticos com padrão de consumo mais intensivo.

Por essa razão, é importante desenvolver estudos que levem em conta o padrão de consumo e a estrutura doméstica também em países em desenvolvimento, uma vez que seria possível identificar com maior clareza a tendência de um padrão de consumo mais intensivo em domicílios menores. O trabalho de Rodrigues (2009) aponta para essa relação, a partir da Pesquisa de Orçamentos Familiares (POF), e acena para uma melhor compreensão dos fatores relacionados ao consumo doméstico e a redução do tamanho dos domicílios, sobretudo pelo declínio do número médio de filhos no caso brasileiro.

Como consequência, vale pensar também na relação de consumo que pode ser atribuída pela estrutura etária da população e tem uma contribuição fundamental. Uma população com uma razão de dependência de jovens (pessoas com menos de 15 anos/ pessoas de 15 a 64 anos) de $75 \%$, como era 
o Brasil de 1950 (CARVALHO; WONG, 2008), deverá apresentar um padrão de consumo menor do que uma população em que essa mesma razão seja da ordem de $28 \%$, como é estimado para o Brasil em 2050.

A razão de dependência de um país costuma associar a relação de emprego/ renda entre a população economicamente ativa e os idosos e jovens (que teoricamente não contribuem com ingressos de renda), sem considerar, normalmente, a dimensão do consumo. Mas parece evidente que haja uma relação direta entre a redução da razão de dependência e o aumento do padrão de emissões de GEE decorrentes de um crescimento de consumo. O trabalho de Jiang e Hardee (2009) aponta as diferenças entre os padrões de consumo encontrados para diferentes grupos etários, devido às suas características específicas. Portanto, diferenciais na proporção de pessoas em cada grupo etário terão impacto diferenciado nas emissões de GEE.

Enfim, as evidências indicam que existem mais impactos provenientes da dinâmica demográfica do que apenas o seu crescimento absoluto, especialmente num contexto em que, a despeito das projeções utilizadas pelo IPCC nos cenários $\mathrm{B} 2$ e A2, pode-se notar que, a cada nova revisão das projeções feitas pelas Nações Unidas, a tendência de decréscimo populacional até o fim do século parece mais provável. Martine (2009), mencionando o trabalho de Jiang e Hardee (2009), salienta que o envelhecimento populacional também parece ser um fator importante, embora menos consistente devido aos impactos de outras variáveis, como o desenvolvimento tecnológico e a compensação que é dada pelo aumento da razão de dependência.

Enfim, há que se pensar no potencial analítico da demografia, mas que tenha a mudança climática como pano de fundo da discussão. Não se trata de uma demografia do meio ambiente, que difere em muito do que poderia vir a ser uma demografia ambiental. Trata-se de incorporar as análises demográficas e de seus componentes de forma integrada para se pensar nas relações que essas mudanças podem ter no padrão futuro de emissões de GEE. Mas isso só será possível com a união de esforços das diversas áreas de pesquisa e não apenas dos "ambientalistas".

As evidências recentes das pesquisas apontam para um potencial enorme da análise demográfica para a melhor compreensão dos padrões de emissões de GEE. Embora ainda haja muito a se fazer, os estudos sobre populações em situação de risco, vulnerabilidade e adaptação permitiram avanços importantes nas análises demográficas que se relacionam com as mudanças climáticas do futuro (HOGAN, 2005 e 2009; HOGAN; MARANDOLA Jr., 2007; HOGAN; TOLMASQUIM, 2001), mas ainda há muito que se avançar na compreensão dos fatores demográficos que contribuem para o aumento ou não das emissões de GEE. A ausência desse debate, sobretudo nos países em desenvolvimento, oferece o risco de simplificar as relações dinâmicas que existem entre população, tecnologia, desenvolvimento econômico e as mudanças climáticas.

Retomando o exposto na introdução, a mudança climática não trata da natureza, mas sim do modo de vida que deveremos ou pretendemos levar no futuro próximo. Não é porque concordamos que a explosão populacional não é uma ameaça ao meio ambiente que outros aspectos demográficos não devam ser incluídos na contabilidade dos impactos das mudanças climáticas. Pelo contrário, todos os indícios apontam que a transição demográfica trará mais elementos para se pensar nas emissões de GEE do que o crescimento da população.

Enfim, considerando a posição do Brasil no processo de transição demográfica e sua relevância no cenário das políticas ambientais, os esforços empreendidos para a meIhor compreensão dessas relações poderá contribuir no entendimento dos processos demográficos e ambientais que ainda se darão nos países em desenvolvimento. 0 desafio é grande, sobretudo para o diálogo interdisciplinar, mas o caminho parece ser inevitável. Como visto, já existem esforços importantes nesse sentido, mas ainda deve-se investir mais em pesquisas que "encontrem" a dimensão ambiental das mudanças na dinâmica demográfica. 


\section{Referências}

BARBIERI, A. F.; DOMINGUES, E.; QUEIROZ, B. L.; RUIZ, R. M.; RIGOTI, J. I.; CARVALHO, J. A. M.; RESENDE, M. F. Climate change and population migration in Brazil's Northeast: scenarios for 2025 2050. Population and Environment, v. 31, p. 344-370, 2010.

BUTTEL, F. H.; DICKENS, P.; DUNLAP, R. E.; GIJSWIJT, A. Sociological theory and the environmentt: an overview and introduction. In: BUTTEL, F.H. et al. (Eds.). Sociological theory and the environment: classical foundations, contemporary insights. Lanham: Rowman and Littlefield Publishers, 2002.

CARMO, R. L.; SILVA, C. A. M. População e mudanças climáticas no contexto litorâneo: uma análise na Região Metropolitana da Baixada Santista. Revista VeraCidade, v. 4 , p. $6,2009$.

CARVALHO, J. A. M. Crescimento populacional e estrutura demográfica no Brasil. Belo Horizonte: Cedeplar, 2004.

CARVALHO, J. A. M.; GARCIA, R. A. O envelhecimento da população brasileira: um enfoque demográfico. Cadernos de Saúde Pública, Rio de Janeiro, v.19, n.3, p. 725-733, maio-jun. 2003.

CARVALHO, J. A. M.; WONG, L. L. R. A transição da estrutura etária da população brasileira na primeira metade do século XXI. Cadernos de Saúde Pública, Rio de Janeiro, v. 24, n. 3, p. 597-605, mar. 2008.

CONFALONIERI, U. E. C.; MARINHO, D. P.; RODRIGUEZ, R. Public health vulnerability to climate change in Brazil. Climate Research, v. 40, p. $175-186,2009$.

CURRAN, S. R.; DE SHERBININ, A. Completing the picture: the challenges of bringing "consumption" into de populationenvironment equation. Population and Environment, v. 26, n. 2, p. 107-131, Nov. 2004.

DODMAN, D. Urban form, greenhouse gas emissions and climate vulnerability. In: GUZMAN, J. M.; MARTINE, G.; McGRANAHAN, G.; SCHENSUL, D.; TACOLI, C. (Eds.). Population dynamics and climate change. New York/London: UNFPA/IIED, 2009, p. 64-79.

EWING, R. et al. Growing cooler: the evidence on urban development and climate change. Washington: Urban Land Institute, 2008.

GIDDENS, A. A política da mudança climática. Rio de Janeiro: Zahar, 2010.

HOBSBAWN, E. Era dos extremos: o breve século XX 1914-1991. São Paulo: Companhia das Letras, 1994.

HOGAN, D. J. Mobilidade populacional, sustentabilidade ambiental e vulnerabilidade social. Revista Brasileira de Estudos de População, v. 22, n. 2, p. 232-338, 2005.

População e mudanças ambientais globais. In: HOGAN, D. J.; MARANDOLA Jr., E. (Orgs.). População e mudança climática: dimensões humanas das mudanças ambientais globais. Campinas: Nepo/ UNFPA, 2009, p. 11-24.

HOGAN, D. J.; MARANDOLA Jr., E. Towards an interdisciplinary conceptualization of vulnerability. Population, Space and Place, Inglaterra, n.11, p.455-471, Oct. 2005.

. Vulnerability to natural hazards in population-enviroment studies. New York: Population and Environment Research Network (PERN), 2007. [Background paper to the PERN cyberseminar on Population \& Vulnerability].

(Orgs.). População e mudança climática: dimensões humanas das mudanças ambientais globais. Campinas: Nepo/ UNFPA, 2009.

HOGAN, D. J.; TOLMASQUIM, M. (Eds.). Human dimensions of global environmental change: brazilian perspectives. Rio de Janeiro: Academia Brasileira de Ciências, 2001.

INTERGOVERNMENTAL PANEL ON CLIMATE CHANGE (IPCC). IPCC special report on emission scenarios. Nebojsa Nakicenovic and Rob Swart (eds.). Cambridge: Cambridge University Press, United Kingdom, 2000. 
Summary for policymakers. Climate change 2007: the physical science basis. Contribution of Working Group I to the fourth assessment report of the intergovernmental panel on climate change. S. Solomon; D. Qin; M. Manning; Z. Chen; M. Marquis; K. B. Averyt; M. Tignor; H. L. Miller (eds.). Cambridge: Cambridge University Press, United Kingdom and New York, 2007a.

Summary for policymakers. Climate change 2007: impacts, adaptation and vulnerability. Contribution of Working Group II to the fourth assessment report of the intergovernmental panel on climate change. M. L. Parry; O. F. Canziani; J. P. Palutikof; P. J. van der Linden; C. E. Hanson (eds.). Cambridge: Cambridge University Press, UK, 2007b, p. 7-22.

Summary for policymakers. Climate change 2007: mitigation. Contribution of Working Group III to the fourth assessment report of the intergovernmental panel on climate change. B. Metz; O. R. Davidson; P. R. Bosch; R. Dave; L. A. Meyer (eds.). Cambridge: Cambridge University Press, United Kingdom and New York, 2007c.

Summary for policymakers. Climate change 2007: synthesis report of the intergovernamental panel on climate change, fourth assessment report. Cambridge: Cambridge University Press, UK, 2007d.

JIANG, L.; HARDEE, K. How do recent population trends matter to climate change? Washington, D.C.: Population Action International, 2009 (Working paper, n. I).

KLEIN, R. J. T. et al. Portfolio screening to support the mainstreaming of adaptation to climate change into development assistance. Climatic Change, v. 84, n. 1, p. 23-44, 2007.

LUTZ, W.; PRSKAWETZ, A.; SANDERSON, W. C. (Eds.). Population and environment: methods of analysis. Population and Development Review, New York, v. 28, Suppl., 2002.

McLEMAN, R. Impacts of population change on vulnerability and the capacity to adapt to climate change and variability: a typology based on lessons from "a hard country". Population and Environment, v. 31, n. 5, p. 286-316, 2009.

McKELLAR, F. L.; LUTZ, W.; PRINZ, C.; GOUJON, A. Population, households and $\mathrm{CO} 2$ emissions. Population and Development Review, v. 21, n. 4, p. 849865, 1995.

MARANDOLA Jr., E.; HOGAN, D. J. As dimensões da vulnerabilidade. São Paulo em Perspectiva, v. 20, n. 1, p.33-43, 2006.

. Vulnerabilidade do lugar vs. vulnerabilidade sociodemográfica: implicações metodológicas de uma velha questão. Rev. Brasileira de Estudos de População, v. 26, n. 2, p. 161-181, 2009.

MARTINE, G. Population dynamics and policies in the context of global climate change In: GUZMAN, J. M.; MARTINE, G.; McGRANAHAN, G.; SCHENSUL, D.; TACOLI, C. (Eds.). Population dynamics and climate change. New York/London: UNFPA/IIED, 2009, p. 9-30.

MOSER, S. C.; LUERS, A. L. Managing climate risks in California: the need to engage resource managers for successful adaptation to change. Climatic Change, v. 87, Suppl. 1, p. 309-322, 2008.

OJIMA, R. Perspectivas para adaptação frente às mudanças ambientais globais no contexto da urbanização brasileira: cenários para os estudos de população. In: HOGAN, D. J.; MARANDOLA Jr., E. (Orgs.). População e mudança climática: dimensões humanas das mudanças ambientais globais. Campinas: Nepo/Unicamp, 2009, p. 11-24.

OJIMA, R.; HOGAN, D. J. Mobility, urban sprawl and environmental risks in brazilian urban agglomerations: challenges for the urban sustainability in a developed country. In: DE SHERBININ, A. et al. (Eds.). Urban population and environment dynamics in the developing world: case studies and lessons learned. Paris: CICRED, 2009.

O'NEILL, B. C.; MACKELLAR, F. L.; LUTZ, W. Population and climate change. Cambridge: Cambridge University Press, 2001. 
QUEIROZ, B. L.; BARBIERI, A. F. Os potenciais efeitos das mudanças climáticas sobre as condições de vida e a dinâmica populacional no Nordeste brasileiro. In: HOGAN, D. J.; MARANDOLA Jr., E. (Orgs.). População e mudança climática: dimensões humanas das mudanças ambientais globais. Campinas: Nepo/Unicamp, 2009, p.159-186.

REID, S.; SMIT, B.; CALDWELL, W.; BELLIVEAU, S. Vulnerability and adaptation to climate risks in Ontario agriculture. Mitigation and Adaptation Strategies for Global Change, v. 12, n. 4, p. 609-637, 2007.

MOSS, R.; BABIKER, M.; BRINKMAN, S.; CALVO, E.; CARTER, T.; EDMONDS, J.; ELGIZOULI, I.; EMORI, S.; ERDA, L.; HIBBARD, K.; JONES, R.; KAINUMA, M.; KELLEHER, J.; LAMARQUE, J. F.; MANNING, M.; MATTHEWS, B.; MEEHL, J.; MEYER, L.; MITCHELL, J.; NAKICENOVIC, N.; O'NEILL, B.; PICHS, R.; RIAHI, R.; ROSE, S.; RUNCI, P.; STOUFER, R.; VAN VUUREN, D.; WEYANT, J.; WILBANKS, T.; VAN YPERSELE, J. P.; ZUREK, M. Towards new scenarios for analysis of emissions, climate change, impacts, and response strategies. Geneva, Technical Summary. Intergovernmental Panel on Climate Change, 2008.

RODRIGUES, E. S. Menos é mais? Uma análise da relação entre os gastos do domicílio e o número de filhos residentes. Dissertação (Mestrado em Demografia). Belo Horizonte: Cedeplar, 2009 [no prelo].

SATTERSWAITTE, D. The implications of population growth and urbanization for climate change. In: GUZMAN, J. M.; MARTINE, G.; McGRANAHAN, G.; SCHENSUL, D.; TACOLI, C. (Eds.). Population dynamics and climate change. New York/London: UNFPA/IIED, 2009, p. 45-63.

UNITED NATIONS. The world at six billion. Table 1, "World Population From" Year 0 to Stabilization, 1999, p. 5.

World population to 2300 . New York, N.Y.: Population Division, Department of Economic and Social Affairs, United Nations, 2004.

YU, E.; LIU, J. Environmental impacts of divorce. PNAS, v. 104 , n. 51 , p. 20629 20634, Dec. 2007.

ZLOTNIK, H. Does population matter for climate change? In: GUZMAN, J. M.; MARTINE, G.; McGRANAHAN, G.; SCHENSUL, D.; TACOLI, C. (Eds.). Population dynamics and climate change. New York/London: UNFPA/ IIED, 2009, p. 31-44.

\section{Resumen}

Las dimensiones demográficas de los cambios climáticos: escenarios de cambio de clima y tendencias de crecimiento poblacional

El cambio climático es un tema cada vez más presente en el discurso político, económico y social. Es innegable que hoy se ha convertido en una cuestión de la más amplia relevancia para toda la sociedad, incluso, especialmente, para la demografía. Como parte inherente de los escenarios de cambio climático, una mejor comprensión de la dinámica demográfica en ese contexto puede contribuir en mucho a un debate responsable. No obstante, todavía existen lagunas que deben ser cubiertas por dos áreas: las ciencias del clima y los estudios de población. Este artículo busca aportar elementos para trasladar este asunto del ámbito ambientalista a una visión más amplia de lo que podría ser una demografía de los cambios climáticos.

Palabras-clave: Proyecciones y tendencias. Dinámica demográfica. Cambio climático. 


\begin{abstract}
The demographic dimension of climate change: climate change scenarios and population growth trends

Climate change has been ever more present in political, economic and social discourse. It has achieved high relevance for society, especially for demographers. A better understanding of the demographic dynamic inherent to climate change scenarios is required for more conscientious debates. There are gaps, however, to be covered for both climate sciences and population studies. This paper seeks to relocate the subject from the environmental field to a broader arena of what could be a demography of the climate change.
\end{abstract}

Keywords: Projections. Demographic dynamics. Climate change.

Recebido para publicação em 09/03/2011

Aceito para publicação em 08/07/2011 
